\title{
Ewing's Sarcoma as a Second Malignancy in Long-Term Survivors of Childhood Hematologic Malignancies
}

\author{
Fabian Wolpert, ${ }^{1,2}$ Michael A. Grotzer, ${ }^{3}$ Felix Niggli, ${ }^{3}$ Dieter Zimmermann, ${ }^{4}$ \\ Elisabeth Rushing, ${ }^{5}$ and Beata Bode-Lesniewska ${ }^{4}$ \\ ${ }^{1}$ Department of Neurology, University Hospital Zurich, Frauenklinikstrasse 26, 8091 Zurich, Switzerland \\ ${ }^{2}$ Department of Medical Oncology, University Hospital Zurich, Rämistrasse 100, 8091 Zurich, Switzerland \\ ${ }^{3}$ Department of Pediatric Oncology, University Children's Hospital, Zurich, Steinwiesstrasse 75, 8032 Zurich, Switzerland \\ ${ }^{4}$ Institute of Surgical Pathology, University Hospital Zurich, Schmelzbergstrasse 12, 8091 Zurich, Switzerland \\ ${ }^{5}$ Department of Neuropathology, University Hospital Zurich, Schmelzbergstrasse 12, 8091 Zurich, Switzerland
}

Correspondence should be addressed to Beata Bode-Lesniewska; beata.bode@usz.ch

Received 3 April 2016; Accepted 19 June 2016

Academic Editor: Natia Esiashvili

Copyright (C) 2016 Fabian Wolpert et al. This is an open access article distributed under the Creative Commons Attribution License, which permits unrestricted use, distribution, and reproduction in any medium, provided the original work is properly cited.

\begin{abstract}
Modern multimodal treatment has significantly increased survival for patients affected by hematologic malignancies, especially in childhood. Following remission, however, the risk of developing a further malignancy is an important issue. The long-term estimated risk of developing a sarcoma as a secondary malignancy is increased severalfold in comparison to the general population. Ewing's sarcoma family encompasses a group of highly aggressive, undifferentiated, intra- and extraosseous, mesenchymal tumors, caused by several types of translocations usually involving the EWSR1 gene. Translocation associated sarcomas, such as Ewing sarcoma, are only rarely encountered as therapy associated secondary tumors. We describe the clinical course and management of three patients from a single institution with Ewing's sarcoma that followed successfully treated lymphoblastic T-cell leukemia or non-Hodgkin lymphoma. The literature on secondary Ewing's sarcoma is summarized and possible pathogenic mechanisms are critically discussed.
\end{abstract}

\section{Introduction}

Modern multimodal treatment strategies have significantly increased the survival of patients affected by hematologic malignancies, including indolent and aggressive forms of lymphoma and leukemia $[1,2]$. In long-term survivors of childhood cancer, the risk of a second cancer is an important issue [3]. Standard treatment for newly diagnosed malignancies varies according to the phenotype, the line of differentiation, biological markers, and extent of the disease and comprises various combinations of chemotherapy [4], radiotherapy, targeted therapy with monoclonal antibodies, and stem cell transplantation, all of which have been implicated as risk factors for second malignancies. The most common second cancers reported in this population include cancers of the head and neck, melanoma, lung cancer, colon cancer, bladder cancer, renal cancer, Hodgkin disease, leukemia, and
Kaposi sarcoma [5]. Patients who received radiation therapy are more likely to develop sarcomas, breast cancers, and mesothelioma compared with patients who have not received it [5].

Ewing's sarcoma (ES) is an aggressive malignancy occurring as a primary tumor mostly in adolescents and young adults with an incidence of about $1 / 1000000$, male to female ratio being 1,5:1 [6]. Most cases of ESs (80\%) originate in bones, where this entity comprises the second most common primary tumor following osteosarcoma in children and adolescents. Up to $20 \%$ of primary ESs are located extraosseously in soft tissues, affecting not only extremities but also various internal organs [7-10]. ESs are characterized at the molecular level by the presence of a balanced translocation between the EWSR1 gene and a member of the ETS gene family, in at least $80 \%$ of cases the partner gene being the FLI1 gene (translocation $\mathrm{t}(11 ; 22)$ (q24;q12) (EWS-FLI1)) [11]. This molecular 
signature and the clinical characteristics of the patients are typical for the peripheral primitive neuroectodermal tumor (PNET) as well. Accordingly, in the current WHO classification [11], PNET once considered a separate entity is now considered to represent part of the histologic spectrum of the "Ewing family of tumors" $[6,12]$. The presence of the fusion gene containing the EWSR1 gene and a member of the ETS family is the most important tumorigenic factor in ES; however, recent genomewide association studies (GWAS) have demonstrated an association of this tumor with the germline background of the affected patients [13]. These studies provide a plausible explanation for the epidemiological finding of a different incidence of ESs across human populations, for example, the significantly higher incidence in patients of Caucasian compared to African origin.

ESs, in contrast to sarcomas with complex karyotypes and without recurring genetic aberrations such as osteosarcoma, have only rarely been reported to occur secondary to cancer therapy, including multimodal therapy for hematologic malignancies $[7,14,15]$. The aim of this study is to analyze three cases of secondary ES in patients from our institutions with T-cell leukemia or non-Hodgkin lymphoma (NHL) as well as summarize documented similar cases from the literature. In addition, the literature review on Ewing's sarcoma as a secondary cancer is summarized and possible pathogenic mechanisms are critically discussed.

\section{Materials and Methods}

2.1. Patients. Three patients with a diagnosis of a genetically confirmed Ewing sarcoma who were previously treated for leukemia or lymphoma during the period of 1991 to 2014 were identified among 98 Ewing sarcoma patients in the archives of the Institute of Surgical Pathology, University Hospital Zurich, Switzerland. Complete follow-up data were available for all 3 patients, including information regarding tumor site and specific treatment modalities of the primary as well as of the secondary malignancy. The available histopathological slides of all 3 cases were reviewed to confirm the diagnosis of ES. A review of the literature published in PUBMED of previously reported cases of ES following another primary malignancy was performed.

The study has been carried out according to the ethical guidelines of our institution.

2.2. Histology and Immunohistochemistry. Tumor tissue samples were fixed in buffered $4 \%$ formalin and embedded in paraffin. $2 \mu \mathrm{m}$ thick sections were stained with hematoxylin and eosin ( $\mathrm{H} \& \mathrm{E})$ according to standard procedures. Immunohistochemistry (IHC) using the CD99 antibody (dilution 1:25, clone HO36-1.1, Novocastra Lab Ltd) was performed on $2 \mu \mathrm{m}$ thick paraffin sections, using the Ventana Benchmark XT automated staining system (Ventana Medical Systems, Tucson, Arizona).

2.3. Fluorescence In Situ Hybridization (FISH). Fluorescence in situ hybridization (FISH) studies were performed on formalin-fixed, paraffin-embedded, $4 \mu \mathrm{m}$ thick tissue sections as described earlier [16]. Dual color break-apart FISH detecting translocations involving the EWSR1 (22q11) gene was performed using commercial probes (Vysis, Abbott AG, Baar, Switzerland). The SpectrumOrange- and SpectrumGreen-labeled EWSR1 probes covered $500 \mathrm{~kb}$ and $1,100 \mathrm{~kb}$ proximal and distal to the EWSR1 gene. The fluorescence staining was visualized with an Olympus BX61 microscope (Olympus, Volketswil, Switzerland) equipped with DAPI, SpectrumGreen and SpectrumOrange filters. Images were acquired with a CCD camera and processed with the AnalySIS imaging software (Soft Imagining System, Munster, Germany). For the assessment of EWSR1 gene rearrangements at least 50 nonoverlapping tumor nuclei were analyzed. If the sample contained at least $25 \%$ of split red and green signals, the tumor was regarded as translocation positive. The signal was considered as split, when red and green fluorescent spots were separated by at least twice the distance occupied by a single probe [17].

2.4. RNA Extraction and RT-PCR. To detect EWSR1-FLI1 and EWSR1-ERG fusions transcripts, total RNA was extracted form paraffin-embedded tissue as described earlier [18]. Amplification was done in a GeneAmp PCR System 9700 (Applied Biosystems) using an OneStep RT-PCR Kit (Qiagen). The $25 \mu \mathrm{L}$ reactions contained $0.4 \mathrm{mM}$ dNTP, 1x OneStep RT-PCR Buffer, $5 \mu \mathrm{L}$ Q-solution, $1 \mu \mathrm{L}$ enzyme mix (all from Qiagen), and 8 units of RNase OUT (Life Technologies). The primer pairs were as follows: (A) TCCTACAGCCAA GCTCCAAGTC (EWSR1-Ex7b) and ATTCATGTTGGGCTTGCTTTTC (FLI1-Ex9); (B) AGAGTAGCTATGGTCAACAA (EWSR1-Ex7a) and CCCA(TA)GCTCCTCT TCTG (FLI1-Ex6); (C) TCCTACAGCCAAGCTCCAAGTC (EWSR1-Ex7b) and CTCCAGGAGGAACTGCCAAA (ERGEx9).

The reverse transcription was done for 30 minutes at $50^{\circ} \mathrm{C}$, followed by a PCR activation step for 15 minutes at $95^{\circ} \mathrm{C}$ and 40 cycles consisting of 1 minute at $94^{\circ} \mathrm{C}, 1$ minute at $56^{\circ} \mathrm{C}$, and 1 minute at $72^{\circ} \mathrm{C}$ followed by final extension at $72^{\circ} \mathrm{C}$ for 10 minutes. RNA-integrity was tested by running ACTB- and IPO8-specific internal control reactions for each sample.

\section{Results}

3.1. Patients. The basic patient characteristics are summarized in Table 1.

3.1.1. Patient 1. T-cell lymphoblastic non-Hodgkin lymphoma was diagnosed in this male patient at the age of 5 years. He first presented with supraclavicular swelling. Histopathologic examination of the resected lymph node revealed high grade T-cell lymphoblastic non-Hodgkin lymphoma (Figure 1(a); unfortunately, except for the text of the final diagnosis and one H\&E slide no further histopathological details are available). Diagnostic workup including chest and abdominal CT scans revealed no further tumor manifestations. He was treated according to the NHL-BFM 90 therapy (BerlinFrankfurt-Munster), which comprises cyclophosphamide, 
TABLE 1: Clinical data on the patients in the current study.

\begin{tabular}{|c|c|c|c|c|c|c|c|c|c|c|}
\hline \multirow[b]{2}{*}{ Pat. } & \multirow[b]{2}{*}{ Gender } & \multirow{2}{*}{$\begin{array}{c}\text { Age at } \\
\text { Dx } \\
\text { (T-ALL) }\end{array}$} & \multirow[b]{2}{*}{$\begin{array}{l}\text { Age at } \\
\text { Dx (ES) }\end{array}$} & \multirow[b]{2}{*}{$\begin{array}{l}\text { Latency } \\
(y)\end{array}$} & \multirow[b]{2}{*}{ Location of ES } & \multicolumn{5}{|c|}{ Treatment of the primary hematological malignancy } \\
\hline & & & & & & $\begin{array}{c}\text { Anthracyclines, } \\
\text { TPII-Inh }\end{array}$ & Vinca & $\begin{array}{c}\text { Alkylating } \\
\text { agent }\end{array}$ & Antimetabolite & RT \\
\hline 1 & $\mathrm{~m}$ & 5 & 8 & 3 & Paravertebral (L5) & $\mathrm{ADR}$ & None & CPA, IFO & Ara-C & None \\
\hline 2 & $\mathrm{~m}$ & 16 & 21 & 5 & Brain, temporal lobe & DNR & VCR & $\mathrm{CPA}$ & $\begin{array}{l}\text { MTX, Ara-C, } \\
\text { 6-MP, 6-TG }\end{array}$ & $\begin{array}{l}\text { Whole brain } \\
\text { (12 Gy) }\end{array}$ \\
\hline 3 & $\mathrm{f}$ & 9 & 11 & 2 & Mandible & DNR & VCR & $\mathrm{CPA}$ & $\begin{array}{c}\text { MTX, Ara-C, } \\
\text { 6-MP, 6-TG }\end{array}$ & $\begin{array}{l}\text { Whole body } \\
\text { (12 Gy) }\end{array}$ \\
\hline
\end{tabular}

DNR, daunorubicin; ADR, doxorubicin; VCR, vincristine; CPA, cyclophosphamide; IFO, ifosfamide; MTX, methotrexate; Ara-C, cytarabine; 6-MP, 6mercaptopurine; 6-TG, thioguanine; RT, radiotherapy.

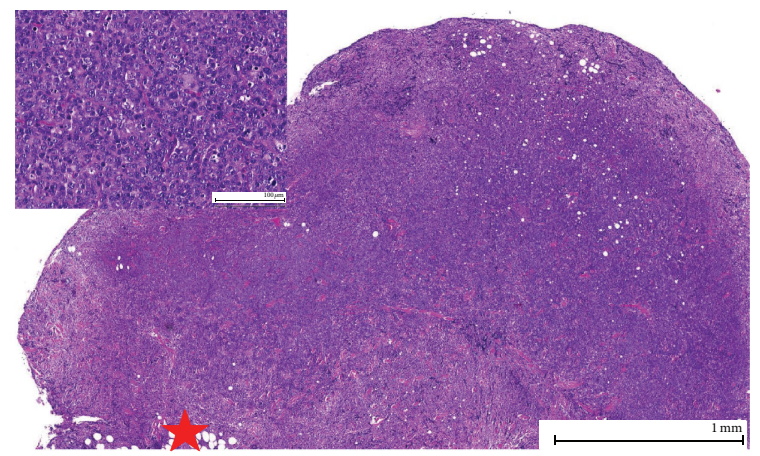

(a)

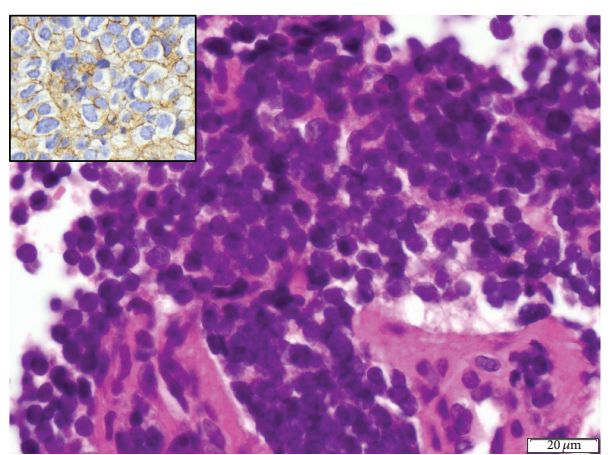

(b)

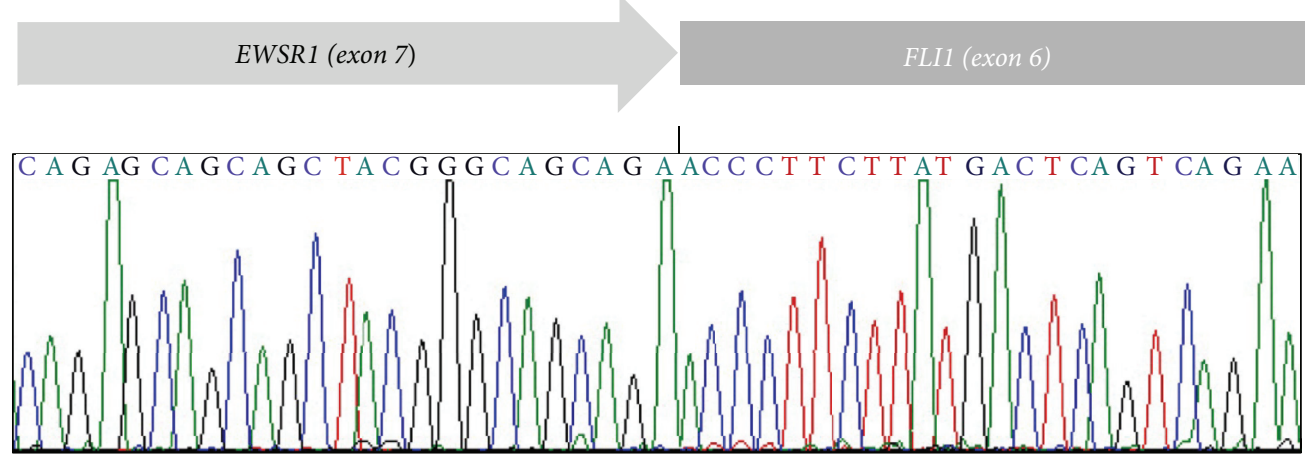

(c)

Figure 1: (a) Biopsy of the cervical mass of patient 1, 3 years prior to current presentation. Diffuse infiltration of the lymph node tissue with effacement of the lymph node architecture and spread to the adjacent adipose tissue (star) by sheets of atypical lymphatic blasts (inset) (H\&E; original magnification 400x). (b) Core biopsy of the paravertebral mass of patient 1 . Solid small, blue round undifferentiated tumor with strong immunohistochemical membranous expression of CD99 (brown reaction product shown in the inset) (H\&E; original magnification 400x). (c) Sanger-sequencing of the RT-PCR amplicon derived from the fusion transcript reveals the most common EWSR1-FLI1 fusion of exon 7 to exon 6, respectively, in the paravertebral tumor tissue of patient 1 confirming the diagnosis of a Ewing sarcoma.

dexamethasone, ifosfamide, methotrexate, cytarabine, prednisolone, etoposide, and cyclophosphamide without radiotherapy. Chemotherapy was completed after 2.5 years in $03 /$ 1998. The patient showed complete remission in the comprehensive follow-up investigations that included laboratory testing, CT scan of brain, chest, and abdomen, bone scan, bone marrow biopsy, cardiac scan, and renal scan.

Three years after the first presentation, he developed nocturnal thigh pain. A bone scan showed a large paravertebral (level L5) mass. Chest and thorax CT revealed lun metastases. Biopsy of the paravertebral lesion showed a malignant small, blue, round, cell tumor, histopathologically consistent with ES (Figure 1(b)). The diagnosis was confirmed by RT-PCR demonstrating the $t(11 ; 22)$ translocation with the EWSR1/FLI1 fusion transcript (Figure 1(c)).

The patient received chemotherapy with vincristine, actinomycin, ifosfamide, adriamycin, carboplatin, epirubicin, and etoposide, based on the CWS-96 protocol (high risk) [19]. Furthermore, he received radiotherapy to the chest and paravertebral region with a cumulative dose of $44.8 \mathrm{~Gy}$. Subsequently, CT scans of the chest, abdomen, and spine revealed complete remission of the pulmonary metastases 


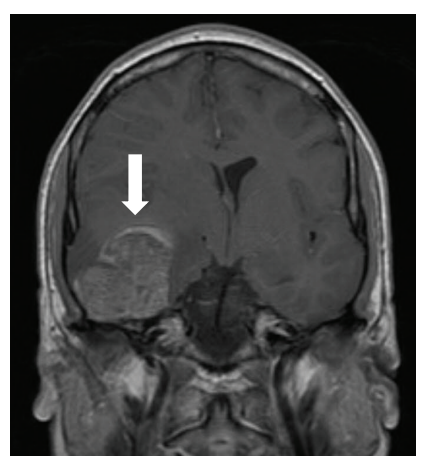

(a)

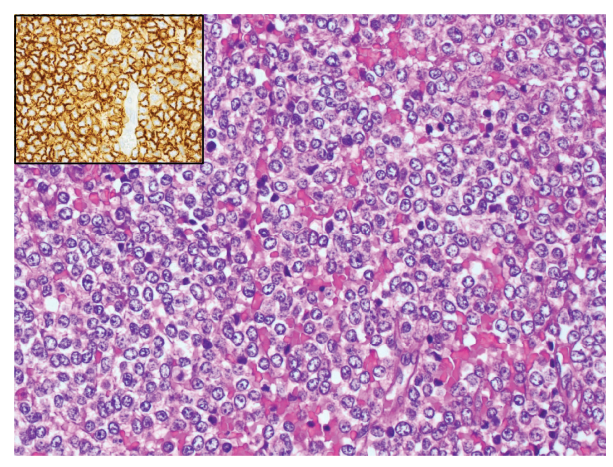

(b)

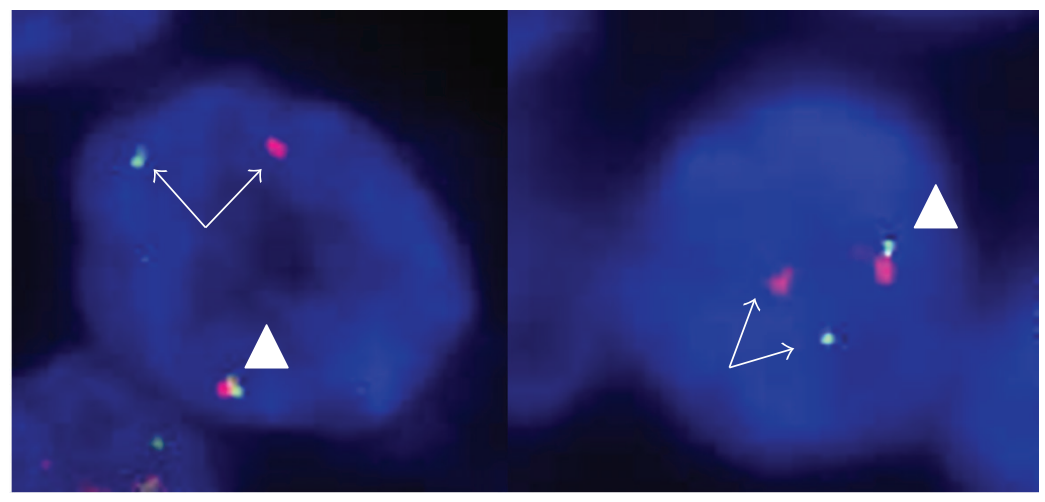

(c)

FIGURE 2: (a) Coronary T1 MRI section demonstrating a $4 \mathrm{~cm}$ mass (arrow) within the right temporal lobe with middle line shift in patient 2. (b) Biopsy of the intracerebral temporal tumor of patient 2 shows solid small, blue round undifferentiated proliferation with a strong immunohistochemical membranous expression of CD99 (brown reaction product shown in the inset) (H\&E; original magnification 200x). (c) Fluorescence in situ hybridization (FISH) of the brain tumor of patient 2, demonstrating the rearrangement of the EWSR1 gene. The nuclei of the tumor cells contain one fused signal (arrow head) and one pair of split green and red signals (arrows).

and size reduction of the left sided paravertebral mass at L4-5 level.

One year later, the patient underwent high dose chemotherapy with busulfan and alkeran and autologous stem cell transplantation. Unfortunately, he developed an infection with an unknown pathogen one month later that proved refractory to antibiotic treatment and he died from acute respiratory distress syndrome.

3.1.2. Patient 2. Acute lymphoblastic T-cell leukemia was diagnosed in this male patient at the age of 16 years. He presented with a mediastinal mass without central nervous system involvement. Bone marrow aspiration showed 95\% blasts infiltration: $\mathrm{CD} 3 \mathrm{c}+$; $\mathrm{CD} 3+$; $\mathrm{CD} 4+$; $\mathrm{CD} 8+$; $\mathrm{CD} 7+$; CD5+; CD34+; CD56+; CD2+; TDT+; TCR a/b+. The patient underwent chemotherapy with vincristine, daunorubicin, L-asparaginase, methotrexate (MTX), and Ara-C. Dexamethasone and thioguanine were given during induction and reinduction. He received whole brain radiation (cumulative dose $12 \mathrm{~Gy}$ ) and 4 doses of intrathecal therapy with MTX, prednisolone, and Ara-C. After completion of therapy in $10 / 2002$, the patient showed hematological remission in the follow-up controls.

Five years and 8 months later, the patient presented to the emergency unit with headache and vomiting. MRI imaging revealed a large intracerebral tumor mass (Figure 2(a)). Resection of the tumor revealed a strongly CD99 positive, small, blue, round cell malignant tumor on histopathological examination (Figure 2(b)) that lacked the expression of lymphatic markers. No rearrangement of the TCR- $\gamma$ gene locus was found on genotyping. The RT-PCR of the fusion products of the two most common Ewing sarcoma translocations $\mathrm{t}(11 ; 22)$ EWSR1/FLI1 and $\mathrm{t}(21 ; 22)$ ESWR1/ERG was negative; however, FISH analysis revealed the EWSR1 gene rearrangement (Figure 2(c)), confirming the diagnosis of Ewing sarcoma and suggesting the presence of one of the rare ES translocation types.

Postoperatively, radiotherapy with $54 \mathrm{~Gy}$ was applied to the right temporoparietal region over 6 weeks. The patient underwent two cycles of cisplatin and CCNU/lomustine; however, chemotherapy had to be discontinued in $02 / 2007$ due to myelotoxicity. At the most recent follow-up in 7/2015, the patient was alive and doing well under anticonvulsive treatment; however, a small mass was found on control MRI in the right frontal lobe, suggesting a metastasis.

3.1.3. Patient 3. This female patient developed acute Tcell leukemia at the age of 9 years. She initially presented with fatigue accompanied by leukocytosis, mediastinal mass, and hepatosplenomegaly, but without CNS involvement. 


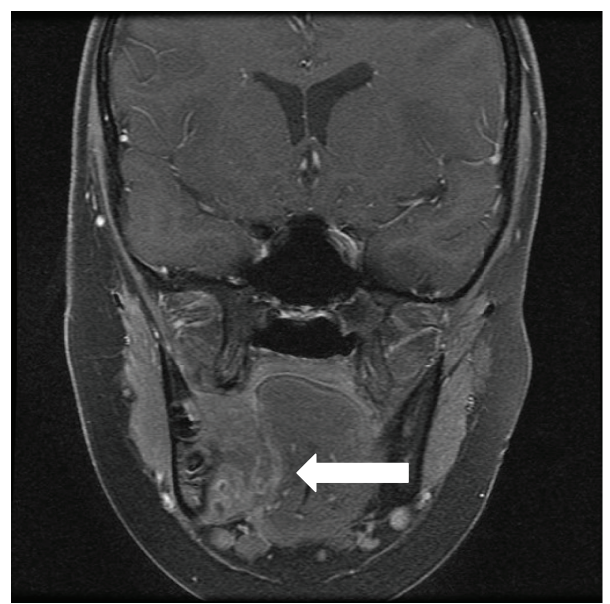

(a)

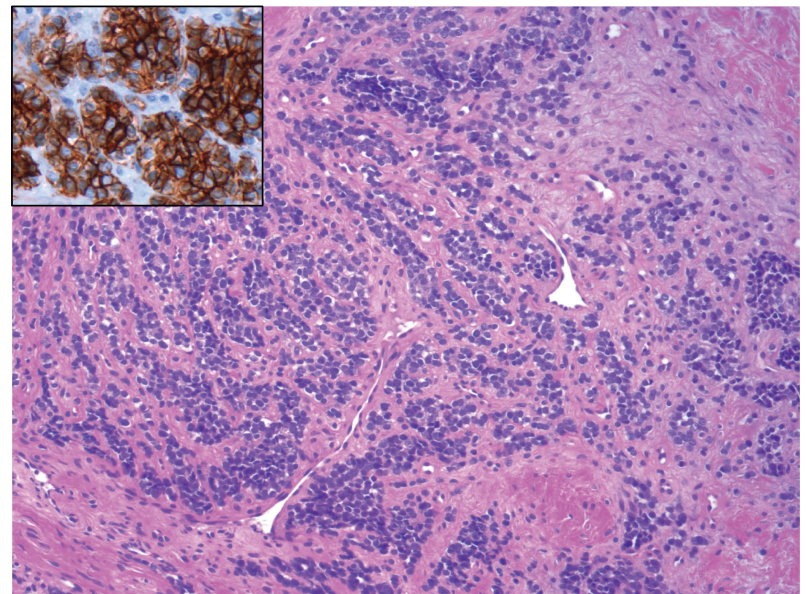

(b)

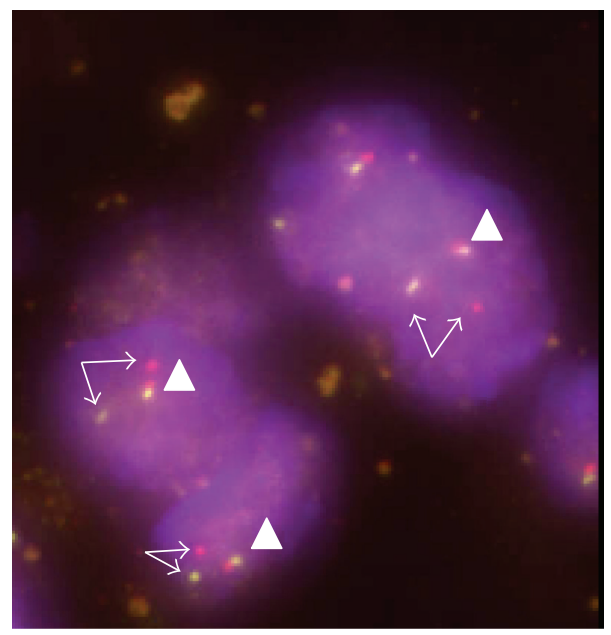

(c)

FIgURE 3: (a) Coronary T1 MRI section demonstrating a large mass (arrow) of the right mandible with infiltration and displacement of the tongue of patient 3. (b) Biopsy of the intraoral mass of patient 3. Diffuse infiltrates of a small, blue round, undifferentiated tumor with strong immunohistochemical membranous expression of CD99 (brown reaction product shown in the inset) (H\&E; original magnification 100x). (c) Fluorescence in situ hybridization (FISH) of the jaw tumor of patient 3, demonstrating the rearrangement of the EWSR1 gene. The nuclei of the tumor cells contain one fused signal (arrow head) and one pair of split green and red signals (arrows).

Cytogenetic analysis of the bone marrow aspiration revealed $\mathrm{t}(1 ; 16)$, loss of TLX3, and biallelic deletion of 12p. The patient was treated according to the high risk protocol of the ALLBFM-2000 trial with prednisone, vincristine, daunorubicin, L-asparaginase, cyclophosphamide, high dose methotrexate, cytarabine, mercaptopurine, thioguanine, and etoposide. Due to the risk stratification an allogenic bone marrow transplantation with an unrelated donor had to be performed five months after the presentation. Conditioning regimen included total body irradiation with a cumulative dose of 12 Gy $(6 \times 2 \mathrm{~Gy})$, etoposide, and antithymocyte globulin Fresenius. A graft versus host disease- (GvHD-) prophylaxis with MTX and cyclosporin A was applied. In the following controls, she showed an enduring remission.

Two years and four months later, the patient reported a swelling in the region of the right 2 nd lower molar tooth. MRI of the head (Figure 3(a)) demonstrated a large tumor mass of the right lower jaw that infiltrated the soft tissues of the floor of the mouth and displaced the tongue. Biopsy revealed the diagnosis of Ewing sarcoma with characteristic small, round, and blue cell histology and typical strong and membranous immunohistochemical expression of CD99 protein (Figure 3(b)). This diagnosis was confirmed by the demonstration of the EWSR1 gene rearrangement on FISH analysis (Figure 3(c)), with the fusion transcript EWSR1/FLI1 of the diagnostic translocation by RT-PCR (the same type of the translocation as in patient 1) (Figure $1(\mathrm{c})$ ). The patient was then treated according to the Euro-Ewing 99 protocol with vincristine, ifosfamide, doxorubicin, etoposide, actinomycin D, and cyclophosphamide. Local tumor control was achieved with total tumor resection and mandibular reconstruction with a fibula plastic. Unfortunately, local ES recurrence with biopsy confirmation was detected one year later and the patient underwent radiotherapy with 
a cumulative dose of $54 \mathrm{~Gy}(30 \times 1,8 \mathrm{~Gy})$ accompanied by second-line chemotherapy (cyclophosphamide, topotecan). Bone metastases in the right femur and fibula were first diagnosed in the following year, followed by wide metastatic spread (spine, lung), which prompted renewed chemotherapy with ifosfamide and palliative mediastinal radiotherapy.

3.2. Review of the Literature. There are only few reports on ES as a secondary malignancy (Table 2), mostly comprising single case reports or short series describing ES following treatment for unrelated tumors, such as breast carcinoma $[20,21]$, retinoblastoma $[22,23]$, or testicular mixed germ cell tumor [24]. The largest series of secondary ES by Applebaum et al. [25] evaluated data from the Surveillance, Epidemiology, and End Results Program (SEER) database between 1973 and 2008 and found 58 cases of secondary ES, accounting for up to $2.1 \%$ of all ES. Only $12.1 \%$ of patients received radiation to the ES site, while the frequency of previous chemotherapy is not reported. Interestingly, five further patients were diagnosed with ES concurrent with other tumors and were therefore not included in the study group of "secondary" ES. This study, however, is purely epidemiological, not including reevaluation of the diagnoses or molecular confirmation of ES. The most common group of primary tumors did not comprise further specified carcinomas (41\%: 24 of 58), with rare cases of non-ES sarcomas, melanomas, brain tumors, or other neoplasia as a the primary tumor. In $24 \%(14 / 58)$ of cases in this study, hematological malignancies preceded secondary ES, representing the second most common primary malignancy group after carcinomas.

The first case of genetically confirmed ES following a chemotherapy malignancy (ALL) was described in 1984 by Tilly et al. [26], although the first case without genetic documentation was mentioned by Meadows et al. [27] in 1977. According to the review of available literature, 38 cases (including the current study) of secondary ES have been described following the diagnosis of a hematologic malignancy (Table 2); however, confirmatory data on genetic tumor background (karyotype, FISH, and/or RT-PCR) has been provided in only 14 cases [14, 26, 28-33].

The diagnosis of secondary ES followed in 1 to 16 years (mean $6 \mathrm{y}$ ) the diagnosis of a hematologic neoplasm, most often an acute lymphoblastic leukemia (46\%: 11 of 24 cases with available information), Hodgkin lymphoma, or an aggressive form of non-Hodgkin lymphoma (5 cases $(20 \%)$ each). There were only two patients with myelogenous neoplasia and 1 with no further specified small cell non-Hodgkin lymphoma. The age of the patients at the first tumor diagnosis ranged from 3 to $22 \mathrm{y}$ (mean $10 \mathrm{y}$ ) and at ES diagnosis from 7 to $27 \mathrm{y}$ (mean $16 \mathrm{y}$ ), with only one substantially older patient (diagnoses at 57 and $65 \mathrm{y}$, resp.). All patients with available data received disease specific chemotherapy for their primary malignancy, consisting of a combination of various groups of cytostatic and cytotoxic substances (Table 2) with no obvious causative association. Secondary ES occurred most commonly in the chest wall and long bones (7 cases each) followed by visceral organs (3), pelvis (2), somatic soft tissue (2), and mandible (1).

\section{Discussion}

Modern multimodal therapy has led to markedly improved long-term survival of cancer patients. Concomitantly, a well-documented increased incidence of malignancies in cancer survivors in comparison to general population has been observed. Most cases of secondary tumors can be attributed to the effects of long-term toxicity of radioand/or chemotherapy; however, the underlying mechanisms of tumorigenesis in this setting remain unclear. In most instances, nonrandom somatic molecular or chromosomal alterations are thought to be involved in the pathogenesis of secondary tumors. Among sarcomas, most therapy associated tumors belong to the category of tumors showing complex karyotypes with mostly nonrecurring genetic aberrations, including undifferentiated pleomorphic sarcoma, osteosarcoma, or rarely malignant peripheral nerve sheath tumors [41]. Therapy associated angiosarcomas show recurrent amplifications of the c-myc gene [42]. Alternatively, sarcomas with simple karyotypes, carrying specific translocations or point mutations, have only rarely been described in the context of secondary, therapy associated tumors [43]. The underlying pathomechanism of therapy-induced malignancies has been elucidated for only selected secondary tumor entities. For example, secondary leukemia following the use of mitoxantrone as an immune modulating drug in patients with multiple sclerosis has been attributed to inhibition of topoisomerase II (TI-II) [44, 45]. TI-II inhibition has been identified as being crucial in the development of the $t(15 ; 17)$ balanced translocation and generation of the PML-RARalpha fusion transcript characteristic for promyelocytic leukemia [46]. Anthracyclines or other drugs inhibiting TI-II are part of therapeutic regimens for hematologic malignancies (Table 2); however, it remains to be elucidated whether these agents play pathogenetically relevant role in the occurrence of ES as a secondary tumor.

It is well known that immunodeficiency after radio-/ chemotherapy and hematopoietic stem cell transplantation are major challenges for survivors of hematological malignancies [47, 48]. Impaired antitumor activity of the innate or adaptive immune system might represent critical factors that predispose to the failure of the system to detect and eliminate newly mutated cells carrying the Ewing sarcoma translocation, thus allowing for the initiation of secondary ES.

Two of the patients described in the current study received radiation therapy, one total body radiation (TBI) and one to the site of the ES occurrence (brain), suggesting a possible role for the radiotherapy in the induction of the subsequent ES. However, among the data in the literature, previous irradiation to the ES site was noted in only $12.1 \%$ of the patients in the study of Applebaum et al. [25] and among the cases compiled in Table 2 with available data on the previous radiation, there were some patients with TBI ( 2 of 7), but also patients with no radiation to the body parts later affected by ES.

The pathogenesis of Ewing sarcoma is strongly linked to the presence of a translocation between the EWSR1 gene and a member of the ETS gene family [11]. The occurrence of the translocation itself was for a long time considered 


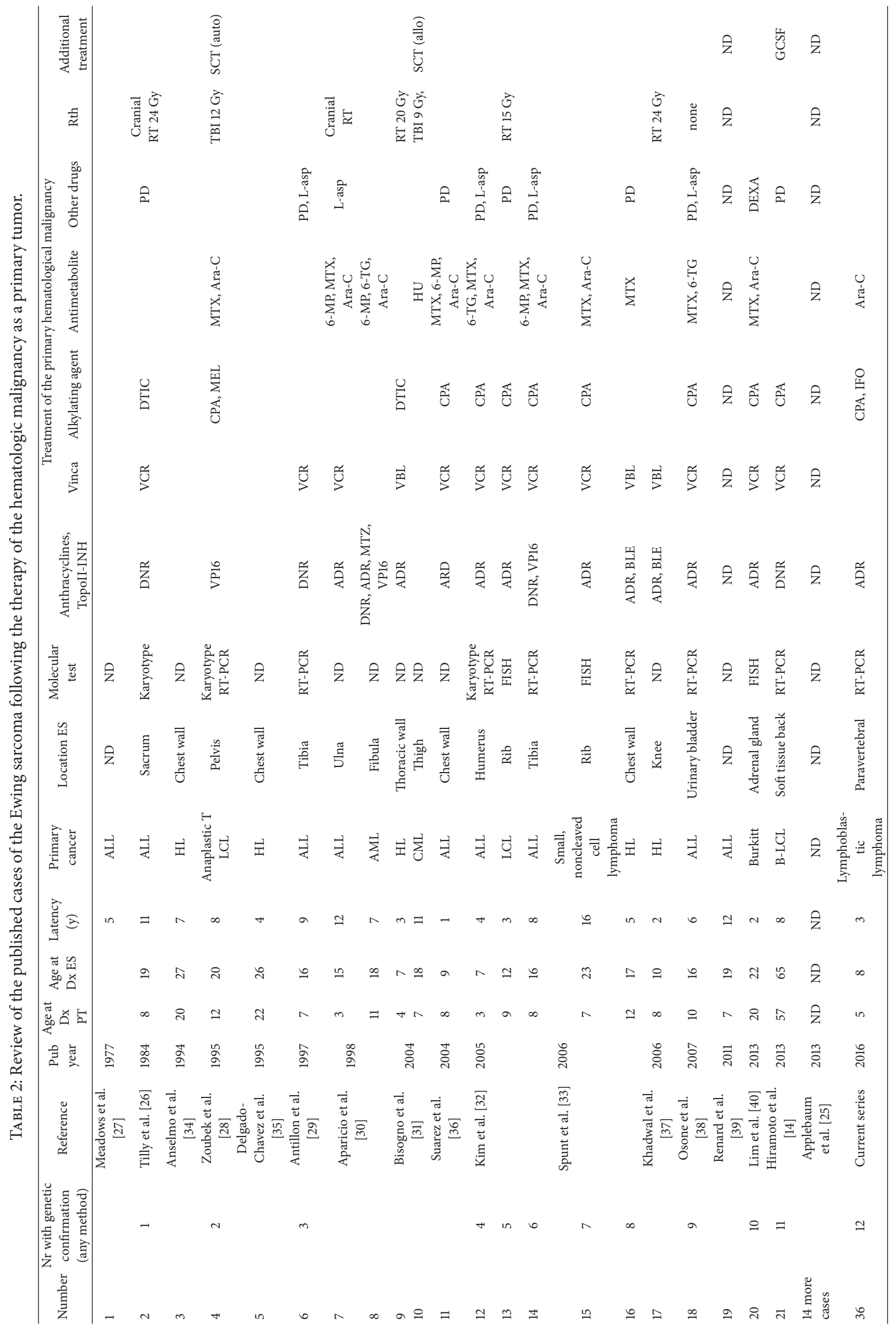




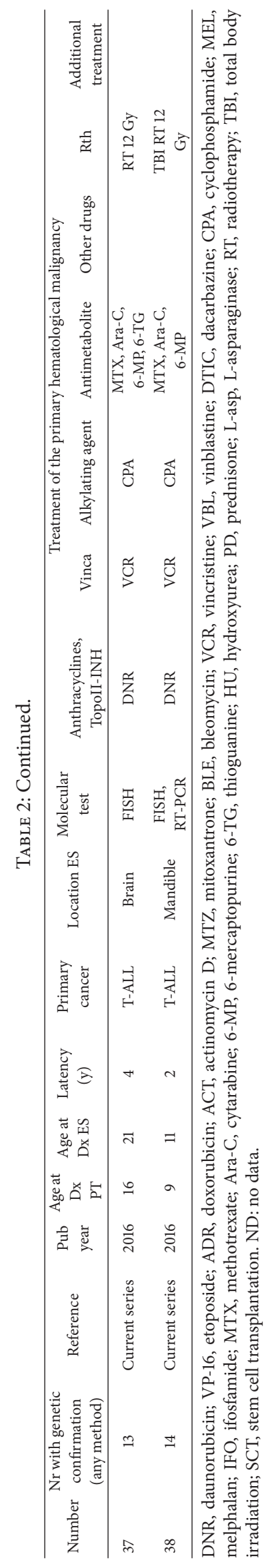


to be a random event. In addition, the risk of developing ES has been linked to increased maternal age [44] and there are associations with exposure to environmental toxins $[3,8,9]$. Alternatively, there are striking differences in the incidence ES according to racial background, ES showing significantly higher incidence among Caucasians than in patients of African origin. Recently published observations [13] of a different frequency of SNPs variants of the EGR2 gene related to Ewing sarcoma susceptibility and may account for this observation, since the frequency of the SNP variant is significantly higher in the non-Africans than in patients of African origin. This important study provides deeper insights into the etiology of the occurrence of this specific translocation associated sarcoma and allows the conclusion that this is not a random disease but rather the expression of a germ line predisposition syndrome, thus accounting for the strong predilection of the Ewing sarcoma to occur in young patients, similar to other genetically linked tumors.

Due to advances in next generation sequencing techniques and the application of powerful computational tools, similar germ line association studies (genomewide association studies, GWAS) are currently possible on large sets of genetic data for many tumor types. Germ line associations of patients with ALL has been recorded for susceptibility, drug response, and toxicities of ALL therapy [49]. Speculatively, as yet not identified common genetic predisposition trait may exist for Ewing sarcoma and some other neoplasia, including hematologic diseases.

Interestingly, in the recent comprehensive study of germ line mutations in the best characterized genes associated with cancer predisposition syndromes in pediatric cancer patients, 5 patients with relevant mutations ( 3 patients with TP53 gene mutations, one patients with PMS2 gene mutation, and one patient with RET gene mutation) were identified among 46 (10.8\%) patients with Ewing sarcoma, frequency of which was higher than in cases of rhabdomyosarcoma $(7 \%, 3 / 43)$ [50]. Ewing sarcoma has been rarely reported in well-defined germ line mutation associated syndromes (Cowden syndrome [51], multiple osteochondromatosis [52], and Williams syndrome [15]); however it has never been listed as a syndrome defining entity. Of note, none of the patients in our study had known cancer predisposition syndromes or family history of a recognized risk constellation.

According to Sultan et al. [53], 3\% of Ewing sarcoma patients develop secondary tumors (4-9-fold risk compared to the general population, the risk being higher for children). In such cases, therapy associated hematologic neoplasms, radiation associated sarcomas, or carcinomas follow multimodal Ewing sarcoma therapy. The relative risk of developing a second malignancy seems to be higher for Ewing sarcoma patients in comparison to other tumors (except for retinoblastoma and primitive neuroectodermal tumors of the central nervous system), suggesting a relationship of ES to either aggressive chemotherapy or genetic predisposition, which may be augmented by the application of cytostatic and cytotoxic drugs.

In summary, we provide a detailed description of welldocumented cases of Ewing sarcoma following a primary hematologic malignancy. Additionally we have reviewed the literature on this association and discussed the possible pathogenetic aspects. As the survival of cancer patients will continue to improve in the future, it is important to understand the risks associated with this success, including the risk of the development of second malignancies, both therapy related and/or linked to the genetic background of the patients.

\section{Competing Interests}

The authors declare that they have no competing interests.

\section{Acknowledgments}

The authors thank José Alves do Carmo, M.D., and Sara Ismail, M.D. (Department of Hematology and Department of Pathology, Hospital Santa Maria, Centro Hospitalar Lisboa Norte, Lisbon, Portugal), for sharing data.

\section{References}

[1] P. C. Nathan, B. Hayes-Lattin, J. J. Sisler, and M. M. Hudson, "Critical issues in transition and survivorship for adolescents and young adults with cancers," Cancer, vol. 117, no. 10, pp. 23352341, 2011.

[2] A. Kishtagari, M. Tavakkoli, and J. H. Park, "Survivorship in adolescents and young adults," Acta Haematologica, vol. 132, no. 3-4, pp. 383-390, 2014.

[3] D. K. Choi, I. Helenowski, and N. Hijiya, "Secondary malignancies in pediatric cancer survivors: perspectives and review of the literature," International Journal of Cancer, vol. 135, no. 8, pp. 1764-1773, 2014.

[4] K. Dunleavy, M. Roschewski, and W. H. Wilson, "Precision treatment of distinct molecular subtypes of diffuse large Bcell lymphoma: ascribing treatment based on the molecular phenotype," Clinical Cancer Research, vol. 20, no. 20, pp. 51825193, 2014.

[5] J. D. Tward, M. M. M. Wendland, D. C. Shrieve, A. Szabo, and D. K. Gaffney, "The risk of secondary malignancies over 30 years after the treatment of non-Hodgkin lymphoma," Cancer, vol. 107, no. 1, pp. 108-115, 2006.

[6] N. Esiashvili, M. Goodman, and R. B. Marcus Jr., "Changes in incidence and survival of Ewing sarcoma patients over the past 3 decades: surveillance epidemiology and end results data," Journal of Pediatric Hematology/Oncology, vol. 30, no. 6, pp. 425-430, 2008.

[7] M. A. Applebaum, J. Worch, K. K. Matthay et al., "Clinical features and outcomes in patients with extraskeletal Ewing sarcoma," Cancer, vol. 117, no. 13, pp. 3027-3032, 2011.

[8] N. J. Balamuth and R. B. Womer, "Ewing's sarcoma," The Lancet Oncology, vol. 11, no. 2, pp. 184-192, 2010.

[9] E.-Y. K. Choi, J. M. Gardner, D. R. Lucas, J. B. McHugh, and R. M. Patel, "Ewing sarcoma," Seminars in Diagnostic Pathology, vol. 31, no. 1, pp. 39-47, 2014.

[10] G. M. Ibrahim, A. Fallah, M. Shahideh, U. Tabori, and J. T. Rutka, "Primary Ewing's sarcoma affecting the central nervous system: a review and proposed prognostic considerations," Journal of Clinical Neuroscience, vol. 19, no. 2, pp. 203-209, 2012.

[11] E. de Alava, L. S. Lessnik, and P. H. Sorensen, "Ewing sarcoma," in WHO Classification of Tumours of Soft Tissue and Bone, C. D. 
M. Fletcher, J. A. Bridge, P. C. W. Hogendoorn, and F. Mertens, Eds., pp. 305-309, IARC, Lyon, France, 2013.

[12] K. N. Ganjoo and S. Patel, "The treatment outcome for adult patients with Ewing's sarcoma," Current Oncology Reports, vol. 15, no. 4, pp. 372-377, 2013.

[13] T. G. P. Grünewald, V. Bernard, P. Gilardi-Hebenstreit et al., "Chimeric EWSR1-FLI1 regulates the Ewing sarcoma susceptibility gene EGR2 via a GGAA microsatellite," Nature Genetics, vol. 47, no. 9, pp. 1073-1078, 2015.

[14] N. Hiramoto, Y. Kobayashi, J. Nomoto et al., "Ewing sarcoma arising after treatment of diffuse large B-cell lymphoma," Japanese Journal of Clinical Oncology, vol. 43, no. 4, pp. 417-421, 2013.

[15] N. Vanhapiha, S. Knuutila, K. Vettenranta, and O. Lohi, "Burkitt lymphoma and Ewing sarcoma in a child with Williams syndrome," Pediatric Blood and Cancer, vol. 61, no. 10, pp. 18771879, 2014.

[16] B. Bode-Lesniewska, J. Hodler, A. von Hochstetter, L. Guillou, U. Exner, and R. Caduff, "Late solitary bone metastasis of a primary pulmonary synovial sarcoma with SYT-SSX1 translocation type: case report with a long follow-up," Virchows Archiv, vol. 446, no. 3, pp. 310-315, 2005.

[17] U. Yamaguchi, T. Hasegawa, Y. Morimoto et al., "A practical approach to the clinical diagnosis of Ewing's sarcoma/primitive neuroectodermal tumour and other small round cell tumours sharing EWS rearrangement using new fluorescence in situ hybridisation probes for EWSR1 on formalin fixed, paraffin wax embedded tissue," Journal of Clinical Pathology, vol. 58, no. 10, pp. 1051-1056, 2005.

[18] B. Bode, S. Frigerio, S. Behnke et al., "Mutations in the tyrosine kinase domain of the EGFR gene are rare in synovial sarcoma," Modern Pathology, vol. 19, no. 4, pp. 541-547, 2006.

[19] D. Modritz, R. Ladenstein, U. Pötschger et al., "Treatment for soft tissue sarcoma in childhood and adolescence. Austrian results within the CWS 96 study," Wiener Klinische Wochenschrift, vol. 117, no. 5-6, pp. 196-209, 2005.

[20] J. Zhong, N. Chen, X. Chen et al., "Peripheral primitive neuroectodermal tumor of the kidney in a 51-year-old female following breast cancer: a case report and review of the literature," Oncology Letters, vol. 9, no. 1, pp. 108-112, 2015.

[21] M. Cole, S. Parajuli, D. Laske et al., "Peripheral primitive neuroectodermal tumor of the dura in a 51-year-old woman following intensive treatment for breast cancer," American Journal of Case Reports, vol. 15, pp. 294-299, 2014.

[22] K. J. Helton, B. D. Fletcher, L. E. Kun, J. J. Jenkins III, and C. B. Pratt, "Bone tumors other than osteosarcoma after retinoblastoma," Cancer, vol. 71, no. 9, pp. 2847-2853, 1993.

[23] J. U. Cope, M. Tsokos, and R. W. Miller, "Ewing sarcoma and sinonasal neuroectodermal tumors as second malignant tumors after retinoblastoma and other neoplasms," Medical and Pediatric Oncology, vol. 36, no. 2, pp. 290-294, 2001.

[24] S. Tanik, K. Zengin, S. Albayrak, R. Eryilmaz, D. Yilmaz, and N. Pirinçci, "Cutaneous Ewing's sarcoma secondary to chemotherapy given for testis tumor: case report," International Journal of Surgery Case Reports, vol. 5, no. 12, pp. 972-974, 2014.

[25] M. A. Applebaum, R. Goldsby, J. Neuhaus, and S. G. DuBois, "Clinical features and outcomes in patients with secondary Ewing sarcoma," Pediatric Blood and Cancer, vol. 60, no. 4, pp. 611-615, 2013.

[26] H. Tilly, C. Bastard, B. Chevallier, E. Halkin, and M. Monconduit, "Chromosomal abnormalities in secondary Ewing's sarcoma," The Lancet, vol. 324, no. 8406, p. 812, 1984.
[27] A. T. Meadows, G. J. D’Angio, V. Mike et al., "Patterns of second malignant neoplasms in children," Cancer, vol. 40, supplement 4, pp. 1903-1911, 1977.

[28] A. Zoubek, I. Simonitsch, E. R. Panzer-Grümayer et al., "Ewing tumor after treatment of $\mathrm{Ki}-1^{+}$anaplastic large cell lymphoma Therapy-associated secondary neoplasm or unrelated coincidence?" Cancer Genetics and Cytogenetics, vol. 83, no. 1, pp. 5-11, 1995.

[29] F. Antillon, S. C. Kaste, J. J. Jenkins et al., "Primitive neuroectodermal tumor of bone as a second malignant neoplasm in a child previously treated for acute lymphoblastic leukemia," Journal of Pediatric Hematology/Oncology, vol. 19, no. 5, pp. 473476, 1997.

[30] J. Aparicio, A. Segura, J. Montalar, A. Verdeguer, V. Castel, and A. B. Sanchez-Heras, "Letter to the editor: 'secondary cancers after ewing sarcoma and ewing sarcoma as second malignant neoplasm,"' Medical and Pediatric Oncology, vol. 30, no. 4, pp. 259-260, 1998.

[31] G. Bisogno, G. Sotti, Y. Nowicki et al., "Soft tissue sarcoma as a second malignant neoplasm in the pediatric age group," Cancer, vol. 100, no. 8, pp. 1758-1765, 2004.

[32] G. E. Kim, B. Beach, J. M. Gastier-Foster et al., "Ewing sarcoma as a second malignant neoplasm after acute lymphoblastic leukemia," Pediatric Blood \& Cancer, vol. 45, no. 1, pp. 57-59, 2005.

[33] S. L. Spunt, C. Rodriguez-Galindo, C. E. Fuller et al., "Ewing sarcoma-family tumors that arise after treatment of primary childhood cancer," Cancer, vol. 107, no. 1, pp. 201-206, 2006.

[34] A. P. Anselmo, C. Cartoni, A. Pacchiarotti et al., "Peripheral neuroectodermal tumor of the chest (Askin tumor) as secondary neoplasm after Hodgkin's disease: a case report," Annals of Hematology, vol. 68, no. 6, pp. 311-313, 1994.

[35] R. Delgado-Chavez, P. Sobrevilla-Calvo, L. Green-Schneewiss, E. Reynoso-Gomez, and L. Quintanilla-Martinez, "Peripheral neuroectodermal tumor of the chest wall in a patient treated for Hodgkin's disease," Leukemia and Lymphoma, vol. 17, no. 56, pp. 509-513, 1995.

[36] C. R. Suarez, S. J. Bertolone, A. B. Raj, and S. Coventry, "Second malignant neoplasms in childhood acute lymphoblastic leukemia: primitive neuroectodermal tumor of the chest wall with germline p53 mutation as a second malignant neoplasm," American Journal of Hematology, vol. 76, no. 1, pp. 52-56, 2004.

[37] A. Khadwal, G. Biswas, B. Arora, P. A. Kurkure, C. Deshmukh, and V. Shetty, "Primitive neuroectodermal tumor (PNET) as second malignancy after treatment of Hodgkin's disease," Indian Journal of Pediatrics, vol. 73, no. 5, pp. 437-438, 2006.

[38] S. Osone, H. Hosoi, K. Tanaka et al., "A case of a Ewing sarcoma family tumor in the urinary bladder after treatment for acute lymphoblastic leukemia," Journal of Pediatric Hematology/Oncology, vol. 29, no. 12, pp. 841-844, 2007.

[39] M. Renard, S. Suciu, Y. Bertrand et al., "Second neoplasm in children treated in EORTC 58881 trial for acute lymphoblastic malignancies: low incidence of CNS tumours," Pediatric Blood and Cancer, vol. 57, no. 1, pp. 119-125, 2011.

[40] S. H. Lim, J. Y. Lee, J. Y. Lee et al., "Unusual presentation of Ewing sarcoma in the adrenal gland: a secondary malignancy from a survivor of Burkitt lymphoma," Japanese Journal of Clinical Oncology, vol. 43, no. 6, pp. 676-680, 2013.

[41] T. O. Henderson, J. Whitton, M. Stovall et al., "Secondary sarcomas in childhood cancer survivors: a report from the childhood cancer survivor study," Journal of the National Cancer Institute, vol. 99, no. 4, pp. 300-308, 2007. 
[42] J. Manner, B. Radlwimmer, P. Hohenberger et al., "MYC high level gene amplification is a distinctive feature of angiosarcomas after irradiation or chronic lymphedema," The American Journal of Pathology, vol. 176, no. 1, pp. 34-39, 2010.

[43] J.-F. Egger, J.-M. Coindre, J. Benhattar, P. Coucke, and L. Guillou, "Radiation-associated synovial sarcoma: clinicopathologic and molecular analysis of two cases," Modern Pathology, vol. 15, no. 9, pp. 998-1004, 2002.

[44] M. Joannides and D. Grimwade, "Molecular biology of therapyrelated leukaemias," Clinical and Translational Oncology, vol. 12, no. 1, pp. 8-14, 2010.

[45] J. L. Nitiss, “Targeting DNA topoisomerase II in cancer chemotherapy," Nature Reviews Cancer, vol. 9, no. 5, pp. 338-350, 2009.

[46] M. Pendleton, R. H. Lindsey, C. A. Felix, D. Grimwade, and N. Osheroff, “Topoisomerase II and leukemia," Annals of the New York Academy of Sciences, vol. 1310, no. 1, pp. 98-110, 2014.

[47] C. N. Duncan, N. S. Majhail, R. Brazauskas et al., "Long-term survival and late effects among one-year survivors of second allogeneic hematopoietic cell transplantation for relapsed acute leukemia and myelodysplastic syndromes," Biology of Blood and Marrow Transplantation, vol. 21, no. 1, pp. 151-158, 2015.

[48] W. Leung, G. Neale, F. Behm et al., "Deficient innate immunity, thymopoiesis, and gene expression response to radiation in survivors of childhood acute lymphoblastic leukemia," Cancer Epidemiology, vol. 34, no. 3, pp. 303-308, 2010.

[49] T. Moriyama, M. V. Relling, and J. J. Yang, "Inherited genetic variation in childhood acute lymphoblastic leukemia," Blood, vol. 125, no. 26, pp. 3988-3995, 2015.

[50] J. Zhang, M. F. Walsh, G. Wu et al., "Germline mutations in predisposition genes in pediatric cancer," The New England Journal of Medicine, vol. 373, no. 24, pp. 2336-2346, 2015.

[51] M. C. Chandhanayingyong, N. M. Bernthal, P. Ungarreevittaya, S. D. Nelson, S. P. Chawla, and A. S. Singh, "Ewing sarcoma in a patient with Cowden syndrome," Journal of the National Comprehensive Cancer Network, vol. 13, no. 11, pp. 1310-1314, 2015.

[52] P. A. Marrero Barrera and P. V. Marrero Ortiz, "Ewing sarcoma superimposed on a previous osteochondroma in multiple osteochondromatosis," Orthopedics, vol. 37, no. 4, pp. e403e406, 2014.

[53] I. Sultan, R. Rihani, R. Hazin, and C. Rodriguez-Galindo, "Second malignancies in patients with Ewing Sarcoma Family of Tumors: a population-based study," Acta Oncologica, vol. 49, no. 2, pp. 237-244, 2010. 


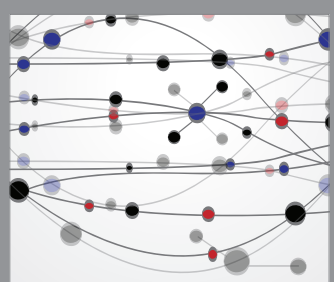

The Scientific World Journal
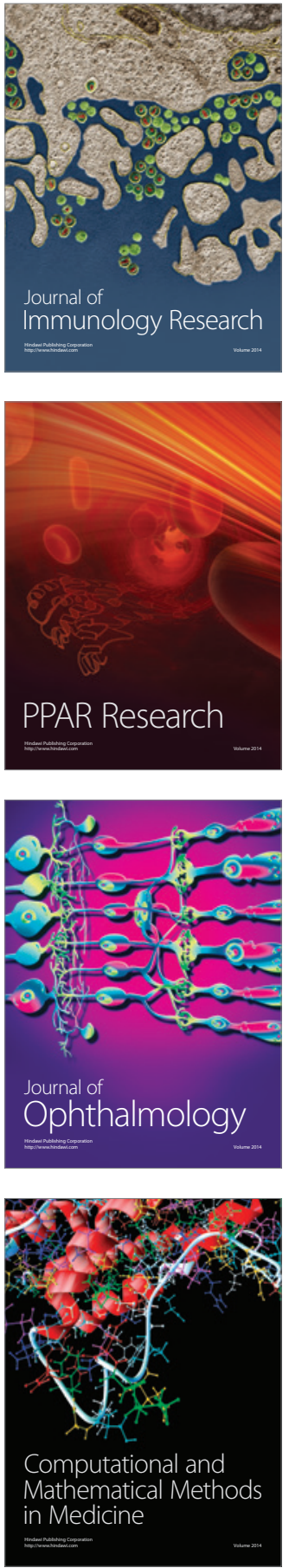

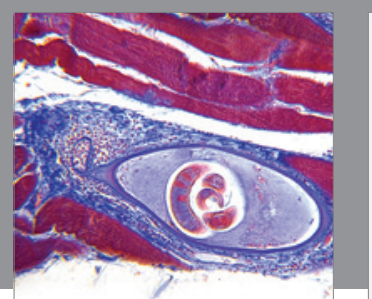

Gastroenterology Research and Practice

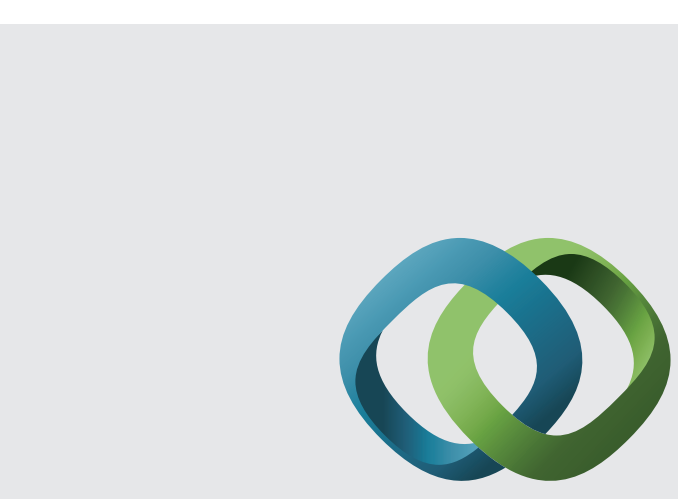

\section{Hindawi}

Submit your manuscripts at

http://www.hindawi.com
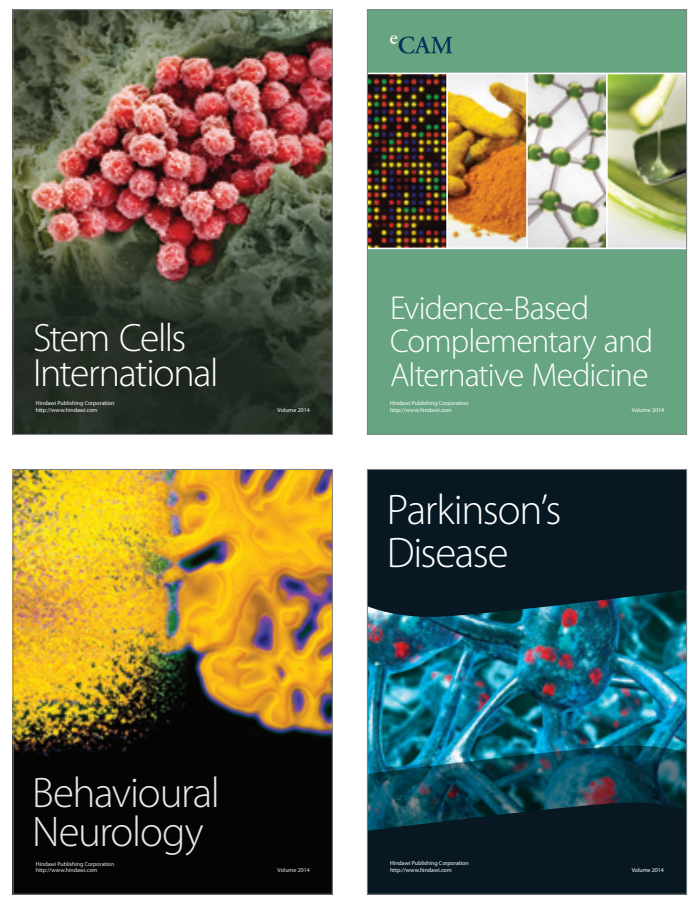
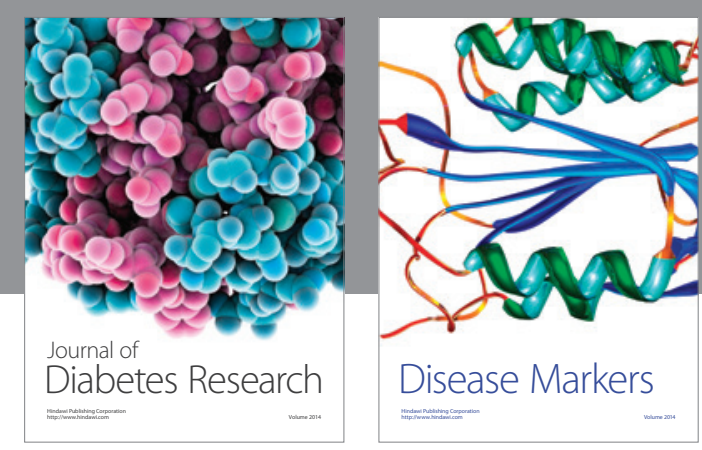

Disease Markers
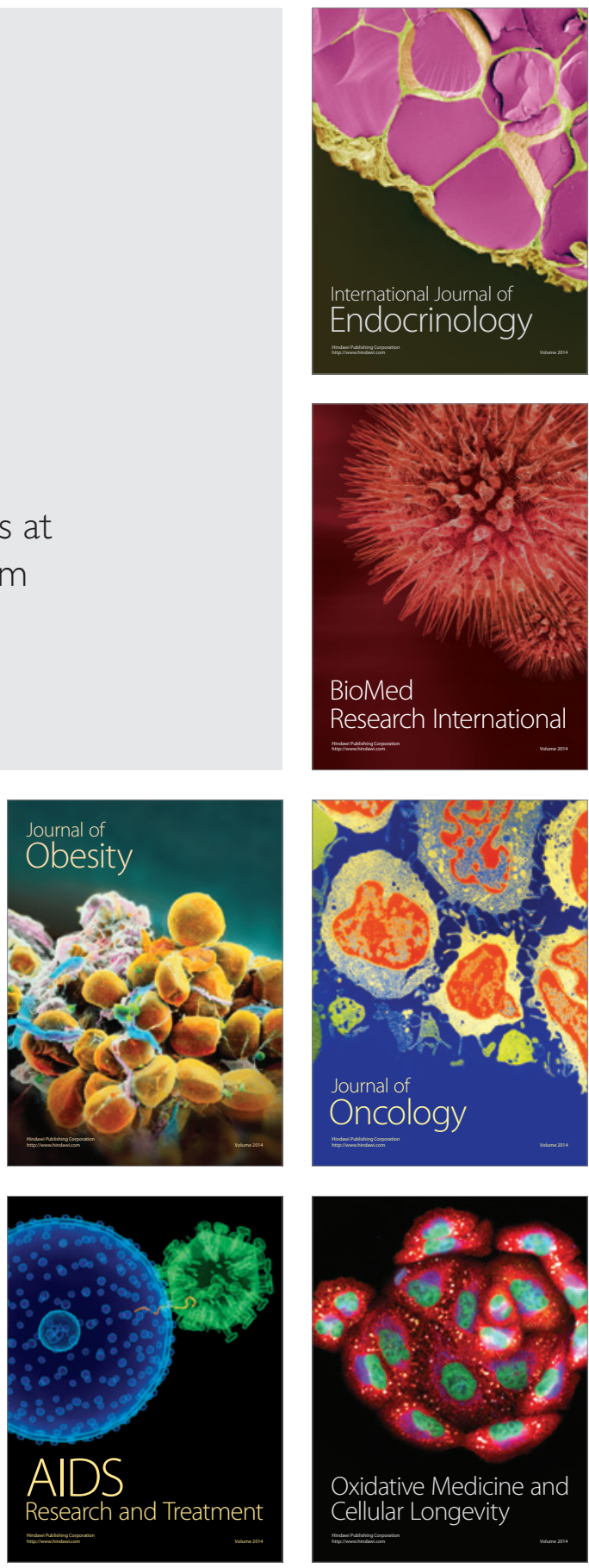